\title{
Decision-making for palliative versus curative intent treatment of retroperitoneal sarcoma (RPS)
}

\author{
Deanna $\mathrm{Ng}^{1,2,3}$, Carol J. Swallow ${ }^{1,2,3}$ \\ ${ }^{1}$ Division of General Surgery, Mount Sinai Hospital, Toronto, ON, Canada; ${ }^{2}$ Department of Surgical Oncology, Princess Margaret Cancer Centre, \\ Toronto, ON, Canada; ${ }^{3}$ Department of Surgery, University of Toronto, Toronto, ON, Canada \\ Contributions: (I) Conception and design: All authors; (II) Administrative support: All authors; (III) Provision of study materials or patients: CJ \\ Swallow; (IV) Collection and assembly of data: D Ng; (V) Data analysis and interpretation: All authors; (VI) Manuscript writing: All authors; (VII) \\ Final approval of manuscript: All authors. \\ Correspondence to: Dr. Carol J. Swallow, MD, PhD. 600 University Avenue, Suite 1225, M5G 1X5, Toronto, ON, Canada.
}

Email: carol.swallow@sinaihealthsystem.ca.

\begin{abstract}
Curative treatment of retroperitoneal sarcoma (RPS) is predicated on complete macroscopic resection, but this is not appropriate in all cases, for a variety of tumour-, patient- and/or treatment-related reasons. Here we review the available literature to glean data that can guide patient selection for major extirpative surgery with its attendant risks or for palliative-intent management that emphasizes quality of life.
\end{abstract}

Keywords: Patient selection; resection; retroperitoneal sarcoma (RPS); tumor biology

Submitted Jul 26, 2018. Accepted for publication Aug 08, 2018.

doi: $10.21037 /$ cco.2018.08.05

View this article at: http://dx.doi.org/10.21037/cco.2018.08.05

\section{Introduction}

For patients with localized primary retroperitoneal sarcoma (RPS), resection remains the cornerstone of treatment and completeness of resection is the most robust predictor of disease specific survival (1-3). Despite this clear correlation, the patient's performance status, and/or extensive involvement of central compartment vasculature may preclude curative-intent resection, even when primary RPS is localized. The decision-making becomes increasingly challenging in the recurrent setting, with diminishing chances of long term disease control and increased potential for perioperative morbidity $(4,5)$. At both primary presentation and recurrence, multifocality and the presence of distant metastases are often viewed as contraindications to resection; nevertheless, the latter may be undertaken in select cases $(6,7)$.

The patient-, tumour- and treatment-related variables that should be scrutinized and weighed in reaching a treatment decision have not been rigorously described or investigated. This is partially due to a literature that until recently consisted largely of single-institution case series with limited patient numbers or population-based data sets that lack key prognostic variables and recurrence data. In the present era of international collaboration, data that can be strategically employed to guide patient selection for resection are becoming available. Here we review the literature regarding outcomes of RPS resection in various challenging scenarios: primary disease that cannot be curatively resected, locally recurrent disease and distant metastases.

\section{Primary disease that cannot be curatively resected}

Oncologic outcome following resection of primary RPS is driven most saliently by the ability to achieve a complete macroscopic resection. Careful examination of good quality cross-sectional imaging and thoughtful preoperative planning will minimize the incidence of grossly incomplete (R2) resection. R2 resection rates vary from $10 \%$ to $20 \%$, centering around $15 \%(8,9)$. R2 resection equates with incurability and such patients have a median survival of 18 months (range 12 to 26 months) (9).

Histologic subtype is also a critical determinant of post-resection recurrence and death from RPS. Well- 
differentiated liposarcoma (WD-LPS) which accounts for $\sim 30 \%$ of RPS is associated with an extremely low rate of distant failure, a moderate rate of local failure at 5 years $(\sim 50 \%)$ but a higher rate by 8 years $(\sim 60 \%)$. Disease specific survival (DSS) is prolonged in these patients at $\sim 85 \%$ at 5 years $(10,11)$. By contrast, leiomyosarcoma (LMS) which accounts for $20 \%$ of RPS, has a relatively low local failure rate, but a high distant failure rate ( $40 \%)$, resulting in a shortened DSS of $\sim 50 \%$ at 5 -year in patients with high-grade LMS (11). The outcome of dedifferentiated (DD) LPS ( $35 \%$ of RPS) varies by grade. Grade 3 DD-LPS patients experience high rates of both distant and local failure ( $40 \%$ and $\sim 30 \%$, respectively at 5 years), with a low rate of 5 -year overall survival (OS) ( 40\%) (10). Grade $1 / 2$ DD-LPS confers a prognosis and pattern of recurrence that are intermediate between those of WD-LPS and grade $3 \mathrm{DD}$ LPS. Knowledge of these recurrence patterns may inform the decision to attempt complete macroscopic resection of primary RPS in the individual patient. That said, even for histologic subtypes with a particularly poor prognosis such as malignant peripheral nerve sheath tumour (MPNST), resection is still favoured, given the lack of other effective modalities.

\section{Principles of curative resection}

Complete gross resection is the goal of surgery, and frequently involves resection of adjacent organs or structures, whether due to frank invasion or intimacy with vasculature. In some series, $\mathrm{R} 1$ resection is associated with inferior outcomes compared with R0 resection. Bremjit et $a l$. at the University of Washington found that patients who had a R0 resection had improved 5 -year OS ( $80 \%$ vs. $30 \%$ in patients with a R1 resection) (12). Similarly, Cho et al. at the Seoul National University Hospital found that patients who had a R0 resection had improved 5-year DSS ( $80 \%$ vs. $50 \%$ in patients with a R1 resection) (13). However, this may reflect differences in margin status according to histologic subtype, which also influences patterns of recurrence (11). In particular, LMS has higher R0 rates, and patients have a significantly lower rate of local recurrence, as noted above. Some centres have advocated so-called compartmental resection in order to achieve higher R0 rates, but convincing evidence of improved control or OS is lacking (14). Abdelfatah et al. at the Johns Hopkins University School of Medicine found no difference in OS ( $60 \%)$ between patients who had a R0/R1 resection (15). Extended multivisceral resection (MVR), especially with pancreas and/or gastric resection, is associated with higher morbidity and mortality $(16,17)$.

\section{Definition of incurability}

Most RPS experts agree that patients with overt distant metastases at the time of primary presentation will not benefit from resection of the primary, as there appears to be no improvement in OS (18). The same is true for multifocal intraabdominal spread, though in some cases there may be a slight survival benefit $(19,20)$. A study by Anaya et al. found that patients with $>7$ discontiguous intraperitoneal/RP tumour deposits had a significantly worse prognosis, with a 5 -year survival rate of $7 \%(6)$.

\section{Technical unresectability}

Unlike locally advanced/borderline resectable pancreatic cancer, there is no current consensus on what constitutes resectability in primary RPS; there is no guide on how to classify tumours as resectable, borderline resectable or upfront technically unresectable (21). Current expert opinions broadly define technical unresectability as the predicted inability to achieve complete macroscopic resection due to extensive tumour involvement of multiple major vascular structures such as the aorta, celiac, superior mesenteric artery (SMA), inferior vena cava (IVC), or due to invasion of the spine with involvement of the spinal cord or extensive involvement of central mediastinal structures (22). Images of a tumour that would unanimously be deemed technically unresectable are shown in Figure 1. This illustrates the case of a 53 -year-old woman who presented with deep-seated abdominal pain. The initial CT scan showed a lobulated mass posterior to the body of the pancreas that involved the splenic vein and bulged into the portal vein near the confluence with the superior mesenteric vein (SMV) (Figure 1A). Percutaneous biopsy revealed a high-grade sarcoma not otherwise specified. A staging CT performed 3 weeks after the first one showed further increase in size of the mass with soft tissue infiltration around the proximal SMA, celiac artery and common hepatic artery as well as progression of the tumour thrombus filling the splenic vein, portal vein, portal vein bifurcation, SMV and tributaries (Figure 1B).

However, the interpretation of imaging findings as in aggregate "unresectable" or "borderline resectable" differs according to the individual surgeon's expertise and attitude. For example, a 53-year-old woman was found to have a grade 2 LMS involving the length of the right ovarian vein, extending into the lumen of the IVC, and intimate with the second part of the duodenum (Figure 2A). After discussion at multi-disciplinary case conference at our centre, this 

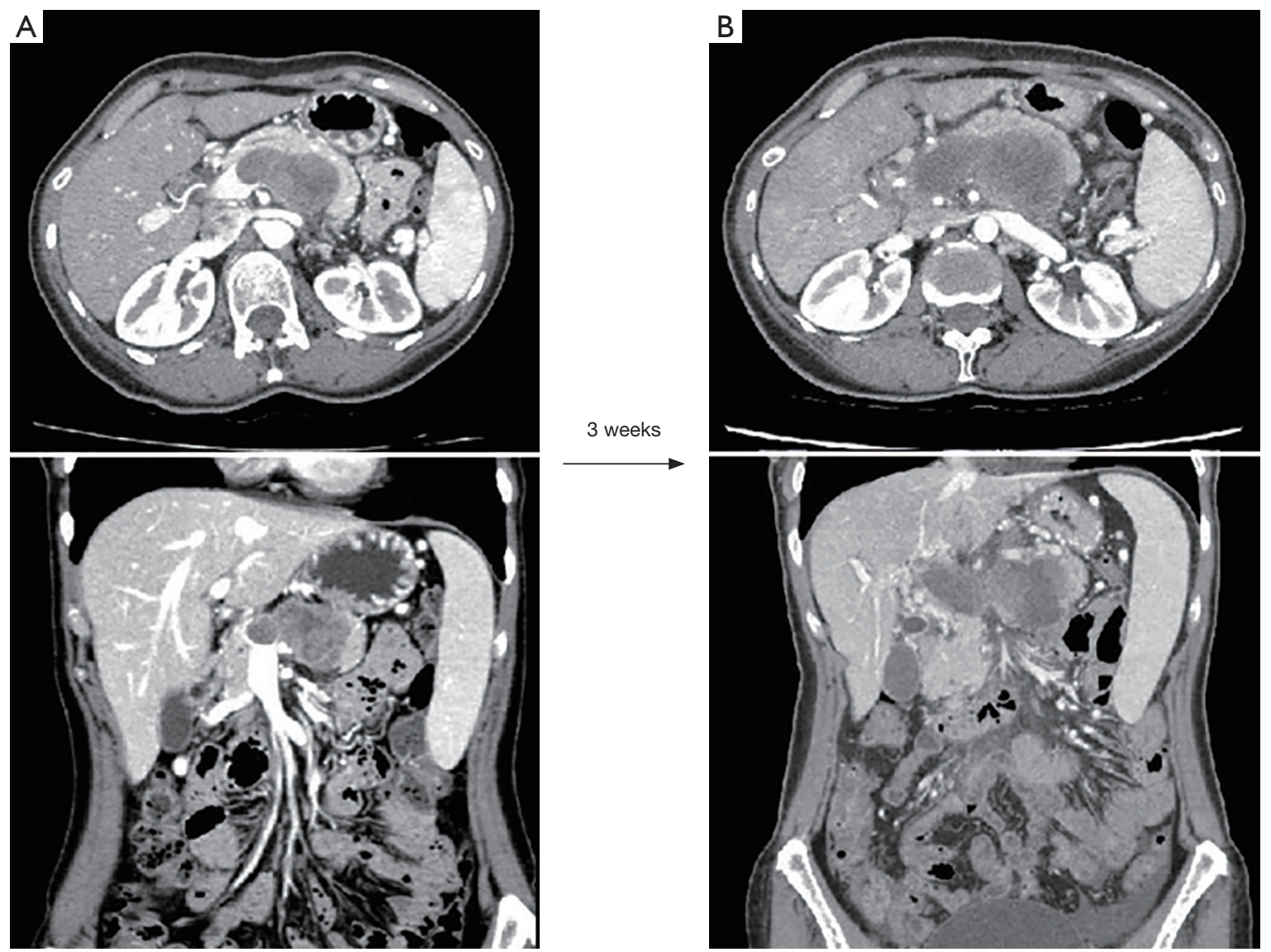

Figure 1 Unresectable high-grade RP sarcoma NOS. (A) Axial and coronal CT scan images at initial presentation of a retroperitoneal solid tumour that fills the splenic vein and extends into the PV-SMV confluence. Percutaneous biopsy showed high-grade sarcoma NOS; (B) restaging CT scan 3 weeks later showed marked progression, encircling the SMA and HA, plus tumour thrombus filling the PV and its bifurcation. RP, retroperitoneal; PV-SMV, portal vein-superior mesenteric vein; NOS, not otherwise specified; HA, hepatic artery.

tumour was classified as "borderline resectable" and the patient received 2 cycles of doxorubicin and ifosfamide; though the overall dimensions of the mass increased on CT scan, there was clear evidence of response with decreased density and vascularity (Figure $2 B$ ).

\section{Adverse tumour biology}

Tumour biology plays a pivotal role in outcomes of resection. This is a catch-all term that encompasses histologic subtype, grade, molecular subtype and unmeasurable parameters unique to the individual tumour and patient. Tumour heterogeneity and clonal development may lead to unexpectedly adverse behaviour. Tumour immunogenicity and the host immune response are also likely of relevance in determining the rate of tumour progression, though to our knowledge, this has not been specifically examined for RPS.
While as discussed, WD-LPS of the RP has a far better prognosis at 5 years than RP DD-LPS, the local failure rate is high at $\sim 50 \%$ at 5 years $(10,23,24)$. This distinguishes WD-LPS of the RP from atypical lipomatous tumour (ALT) of the extremity, suggesting differences in tumour biology that are not apparent from histologic examination.

Within DD-LPS, grade 3 has a worse prognosis than grade $1 / 2(10,11)$. Grade has been incorporated into 3 out of 4 of the RPS specific nomograms as a predictor for survival $(11,25,26)$. Grade may be aligned with tumour biology more so than histology, as in the case of grade 3 DD-LPS and grade 3 LMS, which share similar survival outcomes.

The response to planned preoperative chemotherapy and/or radiotherapy (RT) could potentially inform decisionmaking regarding resection in borderline cases. While any potential benefits of preoperative external beam (XRT) 

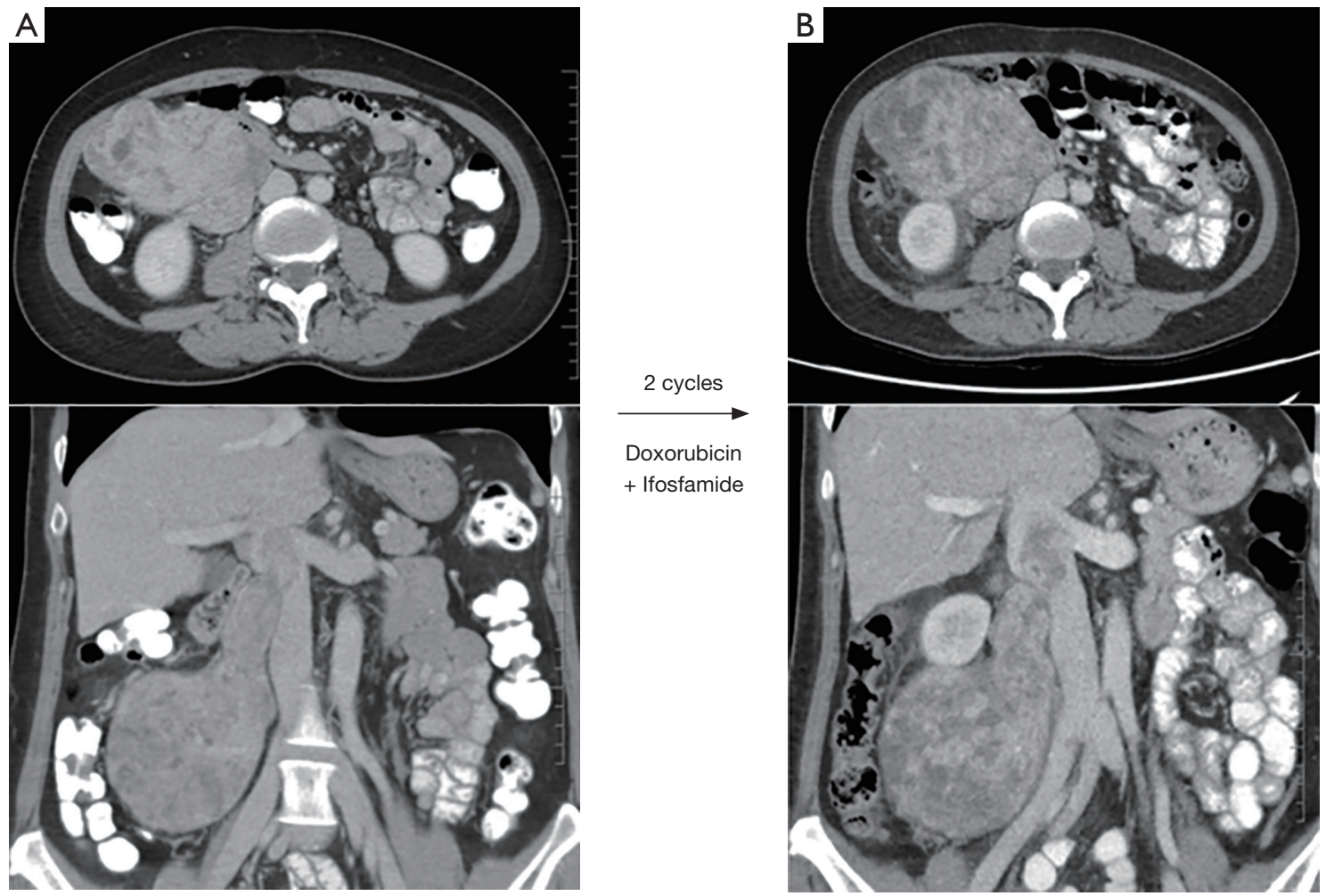

Figure 2 Borderline resectable grade 2 RP LMS. (A) Axial and coronal CT scan images at initial presentation of a retroperitoneal tumour that involves the length of the right ovarian vein, extending into the lumen of the IVC and intimate with the second part of the duodenum; (B) restaging CT scan after 2 cycles of doxorubicin and ifosfamide showed evidence of response to chemotherapy with decreased density and vascularity; however the measured dimensions of the mass increased. RP, retroperitoneal; LMS, leiomyosarcoma; IVC, inferior vena cava.

remain unproven, rates of preoperative XRT have been increasing, with approximately $14 \%$ of American patients now receiving this treatment (27). In some cases, the extent of the primary tumour increases over the course of preoperative XRT, and in some cases DM become apparent. In a combined series of 72 patients who received planned preoperative XRT on a prospective study protocol, investigators from MDACC and University of Toronto found that 10 patients did not come to resection, due to progression of disease and/or decline in PS (28). More recently, a study of spatial and volumetric changes in primary RPS over the course of preoperative RT (29), found that 14 of 19 patients had a decrease in tumour volume, while 5 had an increase in tumour volume; these alterations had no apparent effect on the subsequent quality of surgery, however.

It should be emphasized that there is currently not enough information to indicate how response to treatment should guide decision for resection in primary RPS. The group in Toronto noted that in a cohort of 130 consecutive patients who presented to the Princess Margaret Cancer Centre/Mt Sinai Hospital with primary RPS and no distant metastases on initial cross sectional imaging between January 2012 and April 2017, 10\% (n=4 upfront technically resectable, $n=9$ borderline resectabe) did not undergo resection due to progression of disease. These patients had a median OS of 10 months (range: 1-33). In patients with locally advanced primary RPS and significant comorbidities or compromised PS, a period of observation and reassessment during planned preoperative treatment may allow adverse tumour biology to declare itself. With more data, this strategy could potentially be used as a tool to guide the sometimes-difficult decision for resection.

\section{Patient factors}

Treatment should be individualized according to patient factors. These factors include age, comorbidity index and performance status. Various studies have found increased age to negatively affect prognosis in RPS $(11,30)$. However, 
the relationship between age and survival outcomes can be challenging to understand. When analyzing predictors of OS, age-associated co-morbidities can become confounding variables, and DSS may more accurately reflect any actual independent effect of age on sarcoma recurrence and death from sarcoma. The RPS nomogram developed by the group at MDACC is based on their observation of inferior OS in patients above 65 [hazard ratio (HR) of death: 2.3] (31). Similarly, a nomogram published by a multi-institutional European/North American group (26) reflected a tendency to worse $\mathrm{OS}$ as age increased above 50 . By contrast, data from MSKCC show that age $(<60 v s$. >60) did not predict DM or DSS. Interestingly though, patients under the age of 60 in the latter series had a lower rate of LR (HR: 0.63) (11).

Comorbid conditions and poor performance status are also indicators of poor prognosis in STS patients. A population-based study based in Denmark scored comorbidity according to the Charlson comorbidity index (CCI) in 1,210 patients with extremity or trunk soft tissue sarcoma (32). Patients with $\mathrm{CCI} \geq 1$ had significantly increased overall and disease-specific mortality, even after adjusting for prognostic variables such as age and tumour size. The 5-year overall mortality for patients without comorbidity was $35 \%$, compared to $52 \%, 62 \%$ and $69 \%$ for patients with CCI $=1, \mathrm{CCI}=2$, and CCI $\geq 3$, respectively. The 5 -year disease-specific mortality was $26 \%$ in patients without comorbidity, compared to $33 \%$, $41 \%$ and $44 \%$ in patients with CCI $=1, \mathrm{CCI}=2$, and CCI $\geq 3$ respectively, showing an interplay between comorbidity and death from sarcoma. Indeed, the same Danish group recently analyzed a cohort of 2,167 STS patients (all sites): and found in subset analysis of patients with localized disease that comorbidities negatively affected OS, as might be expected, but also DSS (33).

In general, patients who have worse PS $(\geq 2)$ and limited functional capacity tend to have poorer tolerance for rigorous cancer treatments and this translates to inferior survival outcomes (34). A study by the European Organization for Research and Treatment of Cancer (EORTC)-Soft Tissue and Bone Sarcoma Group (STBSG) and French Sarcoma Group (FSG) found that PS $(\geq 2)$ is the most powerful prognostic factor for 90-day mortality in patients with advanced STS treated with chemotherapy (35). Although this study focused on patients with advanced disease, the findings suggest that the decision for resection should be carefully considered in older patients with significant comorbidities and poor PS. However, more data with regards to comorbidities and performance status has to be collected in RPS patients to provide more robust RPS specific guidelines.

There are few quality of life (QOL) studies that demonstrate how treatment affects patients with RPS. A systematic review of patient reported outcomes following surgery for RPS performed by Olteanu et al. at the University of Toronto (CTOS program, 2017) found that only 26 out of 772 abdominal/RPS studies identified in the world's literature reported on at least one QOL-related outcome. In these 26 studies, QOL was largely grouped by descriptive classifications rather than objective measures, and none of the outcomes was specific to RPS patients. Furthermore, the extent to which health-care teams address the impact of surgery in RPS patients is not known. Covelli et al. at the University of Toronto [2018] investigated the correlation between symptoms experienced 1 year post surgery as self-reported by RPS patients via the Edmonton Symptom Assessment Score (ESAS), and symptoms as recorded in the chart by the health-care team. The results revealed a significant discordance between the two, showing that in the cohort of patients with moderate or severe symptoms recorded via ESAS, $42 \%$ of the time there was no mention of any such symptoms in the clinical notes. Due to the lack of RPS-specific QOL tools, the impact of RPS and its treatment on quality of life may be under-appreciated. In order to facilitate inclusion of QOL considerations in the decision-making for curative intent resection $v s$. palliativeintent management of RPS, more effort must be made to study QOL in these patients.

\section{Palliative resection for primary $R P S$}

Grossly incomplete, or R2, resection of primary RPS is by definition non-curative. In the literature, this is frequently referred to as "palliative", though strictly speaking, the latter term should be reserved for situations where the intent of surgery was to relieve symptoms. R2 resection may be unplanned or planned. When surgery is undertaken with preoperative intent of cure but intraoperative findings preclude complete macroscopic resection, R2 resection is commonly the outcome. Most studies suggest that $\mathrm{R} 2$ resection is associated with limited survival (9). However, in some select cases, such as in large WD-LPS, prolonged survival may be seen even after $\mathrm{R} 2$ resection. A study from MSKCC showed R2 resection was associated with an OS of 24 vs. 12 months for those not resected. Palliative resection for primary RPS in patients who have technically upfront unresectable disease is rarely 
indicated, but may be chosen after careful consideration. This may provide relief of intractable symptoms related to compression of other structures including obstruction and pain, that may compromise quality of life. Such truly palliative intent R2 resections should only be undertaken with the understanding that there is no significant improvement in median survival, and that morbidity of surgery is high $(36,37)$. The subtle decision for an attempt at palliative resection must weigh these limitations against the possibility of temporary symptom relief.

\section{Locally recurrent RPS}

\section{Overview of local recurrence (LR)}

In general, RPS has a high rate of LR compared to extremity STS. In patients with RP LPS, the risk of LR continues unabated past 15, 20 and even 25 years. Postresection, patients most often die from the sequelae of LR without DM (4). Notably, grade 3 DD-LPS carries both high LR and high DM and is associated with particularly poor OS, regardless of treatment (4). Distant metastasis and multifocal abdominal recurrence are generally regarded as contraindications to resection of locally recurrent RPS, given that salvage for cure is extremely unlikely.

\section{Patient selection for resection of $L R$}

The decision for resection of apparently isolated LR of RPS is complex and multifactorial, and should be discussed in a multidisciplinary setting by a group of experienced sarcoma experts. Again, patient-, tumour- and treatment-related variables must be considered. The Transatlantic RPS Working Group (TARPSWG) has developed a consensus document on the approach to management of LR RPS based on large multi-institutional datasets $(4,38)$. This document includes guidance on patient selection for reresection, but ultimately this is a highly individualized and nuanced decision.

Of 1,007 patients with primary RPS described in the original TARPSWG series, all of whom underwent initial resection of the primary, 408 had developed recurrent disease by a median of 23 months; in 219 of these patients, this took the form of an isolated LR. 105 of the 219 underwent resection of the isolated LR, of whom $58 \%$ developed re-recurrence by 5 years (4). Thus, even in this selected group, long term recurrence free survival was frustratingly low. It is not possible to ascertain the criteria that were used across the various participating centres to select patients for resection of LR. However, these would likely relate to putative prognostic factors for survival outcomes in patients who undergo resection for recurrence, as discussed below.

\section{Tumour factors-technical and biologic factors}

As in the primary setting, multifocality portends a poor prognosis, partly because it implies other sites of unrecognized recurrence and also because it is difficult to achieve a complete resection. There is therefore an extremely high likelihood of re-recurrence, some would say that given enough time this rate reaches $100 \%$ (6). Concomitant DM is also an important determinant of outcome in patients with LR. Outcomes are poor in patients with both DM and LR and, as noted, it is generally agreed by experts that only patients with isolated LR should be considered for resection. In the TARPSWG series, 43 of 1,007 patients developed synchronous LR and DM as their first evidence of recurrence. 10 of the 43 underwent resection, and only one patient was re-recurrence free at the time of last follow-up (4).

Disease-free interval (DFI) from primary resection to time of detection of first LR is also correlated with subsequent prognosis. Patients with at least 1 year from time of initial surgery to time of LR tend to do have longer disease free survival (DFS) following resection of LR. Conversely, patients with short DFIs should probably not be offered resection due to the very poor outcomes post resection (DFS at 5 -year $=10 \%$ vs. $60 \%$ with DFI $>1$ year) (5). In fact, a period of observation post recurrence is likely to be of benefit, as the evaluation of growth rate would aid in better patient selection, particularly for LPS (39). A period of observation may also allow for manifestations of DM, particularly relevant in the case of LMS.

Along similar lines, LR of WDLPS is generally more appropriate for resection than LR of DD-LPS. WDLPS is associated with a small chance of DM and extended OS even with multiple local re-recurrences. In a series of patients with recurrent WDLPS, Ikoma et al. found that that DFI $<1$ year and adjacent organ involvement at primary resection predicted inferior OS (40). These tumour characteristics are surrogates for adverse biology. As mentioned, an additional strategy to assess biology is observation of tumour behavior over time via serial imaging. For example, the $1 \mathrm{~cm} / \mathrm{month}$ rule proposed by Singer et al. (41) for assessment of recurrent RP LPS is based on the observation that patients with a growth rate of $<1 \mathrm{~cm} /$ month have better survival (median 
65 vs. 13 months in those with growth $>1 \mathrm{~cm} /$ month). This concept is echoed in the study published recently by Ikoma et al., showing that resection within 6 months of detection of first recurrence was associated with inferior survival (40).

\section{Patient factors}

Even though a site of LR may be of limited size, the extent of the previous resection and the propensity for recurrence at "difficult" sites such as abutting the IVC and/or duodenum and head of pancreas makes resection of LR a potentially morbid procedure. Combined enteric and vascular resections, proximal gastric resection and pancreatic resection all carry a risk of serious lifethreatening complications (13). Patient resilience and potential for recovery are therefore of importance when making the decision for resection of LR. As for resection of primary RPS, these are generally judged by co-morbidity burden, performance status and age, with even greater scrutiny, given the very limited chance of durable disease control. There are at present no specific objective criteria that have been developed to facilitate patient selection for resection of locally recurrent RPS.

\section{Treatment factors}

As emphasized, consideration for resection of LR involves highly individualized decision-making. In addition to the tumour and patient factors mentioned, response to treatment can contribute to the deliberations. We illustrate this point with the following case: a 47 -year-old female initially underwent resection of a primary grade $2 / 3 \mathrm{DD}$ LPS with en bloc right nephrectomy and hemicolectomy. Six years later, local recurrence was managed with XRT (50 Gy total in 25 fractions), then 5 cycles of doxorubicin and ifosfamide. Her LR progressed (Figure $3 A$ ), and she was then started on an MDM2-CDK4 inhibitor, as part of a prospective trial. Over the course of 6 months, her tumour shrank and resection appeared more technically feasible (Figure 3B). Her favourable response to treatment not only enhanced technical resectability but also suggested a relatively favourable biology, and the possibility of a meaningful interval of disease control. In addition, she was very healthy otherwise and had an excellent PS. Reresection was performed. There are several analogies in modern solid tumour management where resection for extensive/recurrent disease is interdigitated with new molecular therapies.

\section{Distant metastasis}

\section{Patterns of distant metastasis}

Distant metastases can be detected at primary presentation, or as a site of recurrence during follow-up after primary resection. Approximately 10-20\% of STS patients are found to have synchronous distant metastases at the time of primary presentation, and $21 \%$ have DM found at the time of recurrence $(7,17)$. Exact rates are not known in patients with RPS. In patients who have had resection of primary RPS and then recur at a distant site, approximately $70 \%$ have DM only while $30 \%$ have both DM and LR. The median time to DM ranges from 12-14 months (4). Tumour biology is extremely important in predicting for DM. Factors that presage for DM include: multifocality of the primary, histologic subtype, grade and in some series, size (11). Most patients who develop DM have LMS or grade 3 DD-LPS, as observed in work by the TARPSWG collaborative as well as in various smaller series. The most common sites of DM are the lung and liver, and rates of metastasis restricted to each of these sites are approximately $20 \%$ and $10 \%$, respectively.

\section{Patient selection for metastectomy}

Prognosis is generally poor in patients with distant metastases, and systemic chemotherapy is for the most part ineffectual in improving overall survival (42). Various small series on metastectomy for isolated hepatic or lung recurrences suggest there may be some overall survival benefit, however, patient selection is clearly an important factor and there is a lack of solid evidence of benefit. The TARPSWG collaborative has published a consensus guide to aid in appropriate selection of patients for various treatment options (43). In general, patients selected to undergo metastectomy should have favourable tumour biology: features suggestive of low volume disease that will allow for a complete $\mathrm{R} 0$ resection, a DFI of $>12$ months, some include recurrent disease that is relatively stable for $>6$ months (with or without systemic therapy). A period of observation following detection of DM would allow for the identification of patients with aggressive tumour biology who would be inappropriate for resection. Importantly, the patient should have good PS with intact pulmonary/hepatic function. There is no role for curative intent resection of multifocal intraabdominal metastases, and this should be considered only in a palliative setting, for only highly selected patients (44). 

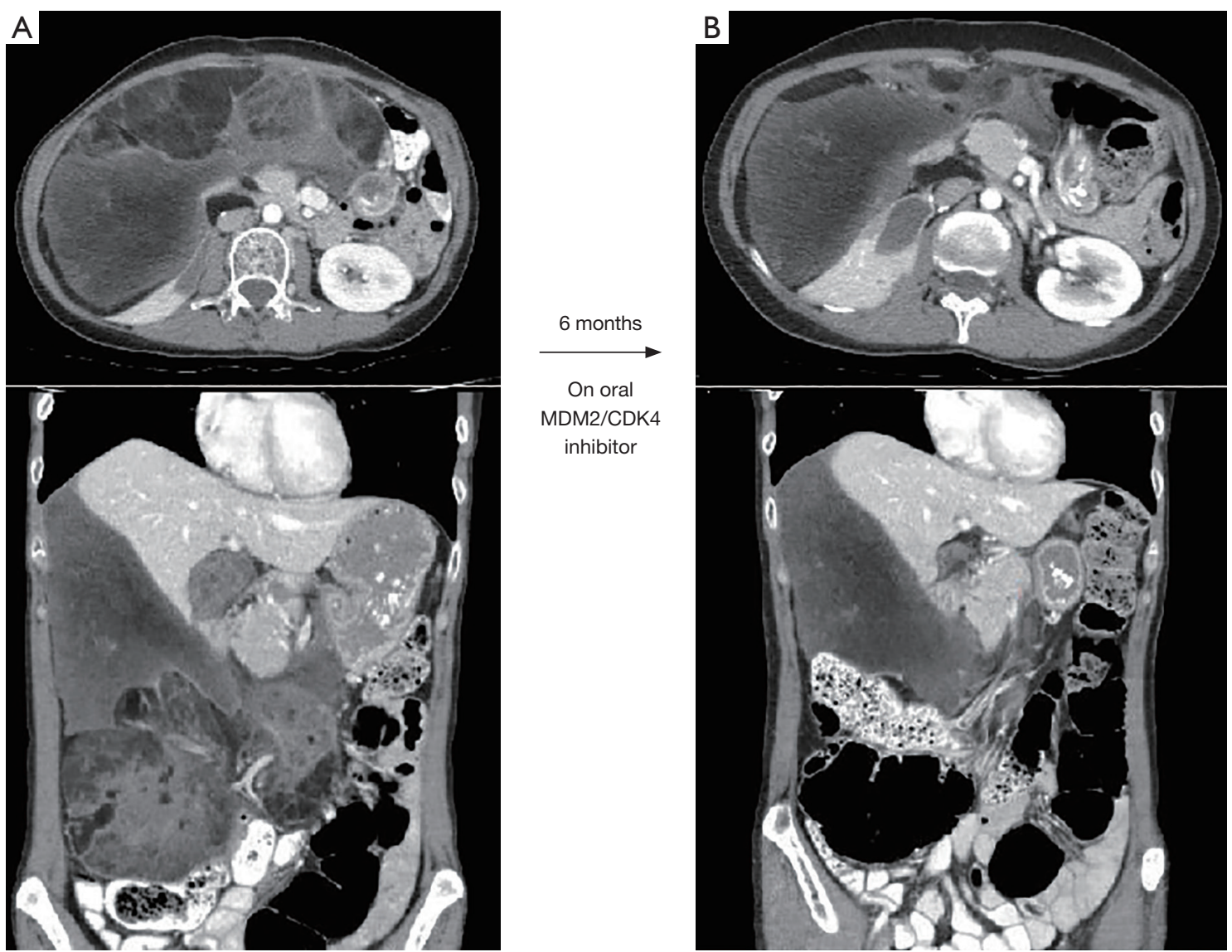

Figure 3 Enhanced resectability of recurrent RP DD-LPS following targeted systemic therapy. (A) Axial and coronal CT scan images showing extent of locally recurrent retroperitoneal DD-LPS following treatment with XRT and cytotoxic chemotherapy; (B) CT scan following 6 months of oral MDM2/CDK4 inhibitor therapy, showing dramatic reduction in extent of tumour, making resection more feasible. RP, retroperitoneal; DD-LPS, dedifferentiated liposarcoma.

\section{Outcomes after metastectomy}

In patients with distant metastases, resection is associated with longer OS (9). Thirty-three case series have been published on patients who have undergone pulmonary resection for isolated pulmonary metastases of STS, with median OS ranging from 32-72 months and 5-year OS ranging from 20-49\% (45). There have been 12 case series published on hepatic resection for isolated hepatic metastases of STS, with median OS ranging from 24-72 months and 5 -year OS ranging from $5.5-45 \%$ (46). These studies show that metastectomy is possible, safe and associated with reasonably prolonged survival in a substantial proportion of highly-selected patients. This is especially true for patients with a longer DFI and with LMS histology (47). In the TARPSWG series, resection was performed in 53 out of the
146 patients who experienced DM. Survival in the group of patients who underwent resection of DM was longer than in patients who did not, with median OS of 29 vs. 21 months and 5 -year OS of $34 \%$ vs. $11 \%$ (4). Further studies that track such patients prospectively are warranted.

\section{Conclusions}

We have discussed the variables that aid selection for resection in various challenging scenarios: primary disease that cannot be curatively resected, locally recurrent disease and distant metastases. Additional data are urgently needed to address the role of response to treatment and patient factors to guide selection for resection. In the present era of international collaboration, we should aim to gather such data in a robust, coordinated fashion. 


\section{Acknowledgements}

None.

\section{Footnote}

Conflicts of Interest: The authors have no conflicts of interest to declare.

\section{References}

1. Keung EZ, Hornick JL, Bertagnolli MM, et al. Predictors of outcomes in patients with primary retroperitoneal dedifferentiated liposarcoma undergoing surgery. J Am Coll Surg 2014;218:206-17.

2. Heslin MJ, Lewis JJ, Nadler E, et al. Prognostic factors associated with long-term survival for retroperitoneal sarcoma: implications for management. J Clin Oncol 1997;15:2832-9.

3. Bonvalot S, Rivoire M, Castaing M, et al. Primary retroperitoneal sarcomas: a multivariate analysis of surgical factors associated with local control. J Clin Oncol 2009;27:31-7.

4. MacNeill AJ, Miceli R, Strauss DC, et al. Postrelapse outcomes after primary extended resection of retroperitoneal sarcoma: a report from the Trans-Atlantic RPS Working Group. Cancer 2017;123:1971-8.

5. Yang JY, Kong SH, Ahn HS, et al. Prognostic factors for reoperation of recurrent retroperitoneal sarcoma: the role of clinicopathological factors other than histologic grade. J Surg Oncol 2015;111:165-72.

6. Anaya DA, Lahat G, Liu J, et al. Multifocality in retroperitoneal sarcoma: a prognostic factor critical to surgical decision-making. Ann Surg 2009;249:137-42.

7. Pawlik TM, Vauthey JN, Abdalla EK, et al. Results of a single-center experience with resection and ablation for sarcoma metastatic to the liver. Arch Surg 2006;141:537-43.

8. Jaques DP, Coit DG, Hajdu SI, et al. Management of primary and recurrent soft-tissue sarcoma of the retroperitoneum. Ann Surg 1990;212:51-9.

9. Zerhouni S, Van Coevorden F, Swallow CJ. The role and outcomes of palliative surgery for retroperitoneal sarcoma. J Surg Oncol 2018;117:105-10.

10. Gronchi A, Strauss DC, Miceli R, et al. Variability in patterns of recurrence after resection of primary retroperitoneal sarcoma (RPS): a report on 1007 patients from the multi-institutional collaborative RPS working group. Ann Surg 2016;263:1002-9.
11. Tan MC, Brennan MF, Kuk D, et al. Histology-based classification predicts pattern of recurrence and improves risk stratification in primary retroperitoneal sarcoma. Ann Surg 2016;263:593-600.

12. Bremjit PJ, Jones RL, Chai X, et al. A contemporary large single-institution evaluation of resected retroperitoneal sarcoma. Ann Surg Oncol 2014;21:2150-8.

13. Cho SY, Moon KC, Cheong MS, et al. Significance of microscopic margin status in completely resected retroperitoneal sarcoma. J Urol 2011;186:59-65.

14. Raut CP, Swallow CJ. Are radical compartmental resections for retroperitoneal sarcomas justified? Ann Surg Oncol 2010;17:1481-4.

15. Abdelfatah E, Guzzetta AA, Nagarajan N, et al. LongTerm Outcomes in Treatment of Retroperitoneal Sarcomas: A 15 Year Single-Institution Evaluation of Prognostic Features. J Surg Oncol 2016;114:56-64.

16. Pisters PW. Resection of some -- but not all -- clinically uninvolved adjacent viscera as part of surgery for retroperitoneal soft tissue sarcomas. J Clin Oncol 2009;27:6-8.

17. Bonvalot S, Miceli R, Berselli M, et al. Aggressive surgery in retroperitoneal soft tissue sarcoma carried out at highvolume centers is safe and is associated with improved local control. Ann Surg Oncol 2010;17:1507-14.

18. Toulmonde M, Bonvalot S, Ray-Coquard I, et al. Retroperitoneal sarcomas: patterns of care in advanced stages, prognostic factors and focus on main histological subtypes: a multicenter analysis of the French Sarcoma Group. Ann Oncol 2014;25:730-34.

19. Chudgar NP, Brennan MF, Munhoz RR, et al. Pulmonary metastasectomy with therapeutic intent for soft-tissue sarcoma. J Thorac Cardiovasc Surg 2017;154:319-30.e1.

20. Billingsley KG, Burt ME, Jara E. Pulmonary metastases from soft tissue sarcoma: analysis of patterns of diseases and postmetastasis survival. Ann Surg 1999;229:602.

21. Lopez NE, Prendergast C, Lowy AM. Borderline resectable pancreatic cancer: definitions and management. World J Gastroenterol 2014;20:10740-51.

22. Greiner RH, Munkel G, Blattmann H, et al. Conformal radiotherapy for unresectable retroperitoneal soft tissue sarcoma. Int J Radiat Oncol Biol Phys 1992;22:333-41.

23. Fairweather M, Wang J, Jo VY, et al. Incidence and adverse prognostic implications of histopathologic organ invasion in primary retroperitoneal sarcoma. J Am Coll Surg 2017;224:876-83.

24. Ikoma N, Roland CL, Torres KE, et al. Concomitant organ resection does not improve outcomes in primary retroperitoneal well-differentiated liposarcoma: A 
retrospective cohort study at a major sarcoma center. J Surg Oncol 2018;117:1188-94.

25. Gronchi A, Miceli R, Shurell E, et al. Outcome prediction in primary resected retroperitoneal soft tissue sarcoma: histology-specific overall survival and disease-free survival nomograms built on major sarcoma center data sets. J Clin Oncol 2013;31:1649-55.

26. Raut CP, Miceli R, Strauss DC, et al. External validation of a multi-institutional retroperitoneal sarcoma nomogram. Cancer 2016;122:1417-24.

27. Nussbaum DP, Speicher PJ, Gulack BC, et al. Long-term Oncologic Outcomes After Neoadjuvant Radiation Therapy for Retroperitoneal Sarcomas. Ann Surg 2015;262:163-70.

28. Pawlik TM, Pisters PW, Mikula L, et al. Long-term results of two prospective trials of preoperative external beam radiotherapy for localized intermediate- or highgrade retroperitoneal soft tissue sarcoma. Ann Surg Oncol 2006;13:508-17.

29. Wong P, Dickie C, Lee D, et al. Spatial and volumetric changes of retroperitoneal sarcomas during pre-operative radiotherapy. Radiother Oncol 2014;112:308-13.

30. Abbott AM, Habermann EB, Parsons HM, et al. Prognosis for primary retroperitoneal sarcoma survivors: a conditional survival analysis. Cancer 2012;118:3321-9.

31. Anaya DA, Lahat G, Wang X, et al. Postoperative nomogram for survival of patients with retroperitoneal sarcoma treated with curative intent. Ann Oncol 2010;21:397-402.

32. Maretty-Nielsen K, Aggerholm-Pedersen N, Safwat A, et al. Prevalence and prognostic impact of comorbidity in soft tissue sarcoma: a population-based cohort study. Acta Oncol 2014;53:1188-96.

33. Raedkjaer M, Maretty-Kongstad K, Baad-Hansen T, et al. The impact of comorbidity on mortality in Danish sarcoma patients from 2000-2013: A nationwide population-based multicentre study. PLoS One 2018;13:e0198933.

34. West HJ, Jin JO. JAMA Oncology Patient Page. Performance Status in Patients With Cancer. JAMA Oncol 2015;1:998.

35. Penel N, Glabbeke MV, Mathoulin-Pelissier S, et al. Performance status is the most powerful risk factor for early death among patients with advanced soft tissue sarcoma: the European Organisation for Research and Treatment of Cancer-Soft Tissue and Bone Sarcoma Group (STBSG) and French Sarcoma Group (FSG) study. Br J Cancer 2011;104:1544-50.

36. Yeh JJ, Singer S, Brennang MF, et al. Effectiveness of palliative procedures for intra-abdominal sarcomas. Ann
Surg Oncol 2005; 12:1084-9.

37. Shiloni E, Szold A, White DE, et al. High-grade retroperitoneal sarcomas: role of an aggressive palliative approach. J Surg Oncol 1993;53:197-203.

38. Trans-Atlantic RPS Working Group. Management of Recurrent Retroperitoneal Sarcoma (RPS) in the Adult: A Consensus Approach from the Trans-Atlantic RPS Working Group. Ann Surg Oncol 2016;23:3531-40.

39. Swallow CJ. Strategic Delay: Histology- and BiologyDriven Decision-Making in Recurrent Retroperitoneal Sarcoma. Ann Surg Oncol 2018;25:2117-9.

40. Ikoma N, Roland CL, Torres KE, et al. Salvage surgery for recurrent retroperitoneal well-differentiated liposarcoma: early reoperation may not provide benefit. Ann Surg Oncol 2018;25:2193-200.

41. Park JO, Qin LX, Prete FP, et al. Predicting outcome by growth rate of locally recurrent retroperitoneal liposarcoma: the one centimeter per month rule. Ann Surg 2009;250:977-82.

42. Savina M, Le Cesne A, Blay JY, et al. Patterns of care and outcomes of patients with METAstatic soft tissue SARComa (STS) in a real-life setting: the METASARC observational study. BMC Med 2017;15:78.

43. Trans-Atlantic Retroperitoneal Sarcoma Working Group (TARPSWG). Management of metastatic retroperitoneal sarcoma: a consensus approach from the Trans-Atlantic Retroperitoneal Sarcoma Working Group (TARPSWG). Ann Oncol 2018;29:857-71.

44. Baratti D, Pennacchioli E, Kusamura S, et al. Peritoneal sarcomatosis: is there a subset of patients who may benefir from cytoreductive surgery and hyperthermic intraperitoneal chemotherapy? Ann Surg Oncol 2010;17:3220-8.

45. Kim S, Ott HC, Wright CD, et al. Pulmonary resection of metastatic sarcoma: prognostic factors associated with improved outcomes. Ann Thorac Surg 2011;92:1780-6.

46. Marudanayagam R, Sandhu B, Perera MT, et al. Liver resection for metastatic soft tissue sarcoma: an analysis of prognostic factors. Eur J Surg Oncol 2011;37:87-92.

47. Lang H, Nussbaum KT, Kaudel P, et al. Hepatic metastases from leiomyosarcoma: a single-center experience with 34 liver resections during a 15 -year period. Ann Surg 2000;231:500-5.

Cite this article as: $\mathrm{Ng} \mathrm{D}$, Swallow CJ. Decision-making for palliative versus curative intent treatment of retroperitoneal sarcoma (RPS). Chin Clin Oncol 2018;7(4):40. doi: 10.21037/ cco.2018.08.05 